\title{
Erasure Coding for Ultra-Low Power Wireless
}

\section{Networks}

\author{
Jalaluddin Qureshi ${ }^{1 *}$, Rizwan Ullah Khan ${ }^{2}$, Chuan Heng Foh ${ }^{3}$, Periklis \\ Chatzimisios $^{4}$ \\ ${ }^{1}$ Department of Computer Science, National University of Computer and \\ Emerging Sciences, Karachi campus, Pakistan \\ ${ }^{2}$ Department of Electrical Engineering, Namal College, Mianwali, Pakistan \\ ${ }^{3} 5 \mathrm{GIC}$, Institute for Communication Systems, University of Surrey, UK \\ ${ }^{4}$ Department of Informatics, Alexander Technological Educational Institute of \\ Thessaloniki (ATEITHE), Greece \\ *Corresponding author jala0001@e.ntu.edu.sg
}

\begin{abstract}
In this paper, we study erasure coding for ultra-low power wireless networks with power consumption in order of milliwatts. We propose sparse parallel concatenated coding (SPCC) scheme, in which we adopt concatenated code over different field sizes so that the total energy cost of the network is minimized. We optimize sparsity and ratio of coded packets over $G F(2)$ (i.e., Galois field of size 2) and larger field size such as $G F(32)$ for different values of $k$. While high sparsity decreases energy cost of encoding, it comes at the tradeoff cost of high reception redundancy, which also results in a larger matrix which the receiver need to invert for decoding. The use of $G F(2)$ packets minimizes the computational cost of encoding and decoding, while the use of small fraction of packets over $G F(32)$ minimizes reception redundancies. Testbed implementation shows that SPCC energy gain increases with increasing packet generation size $k$ compared with the next best performing coding scheme. We show that for the case where $k \leq 40$, SPCC reduces energy cost by up to $100 \%$ compared with the next best performing coding scheme.
\end{abstract}

Keywords: Network Coding; Computational Complexity; Forward Error Correction; Parallel Concatenated Code; Embedded Devices; 


\section{INTRODUCTION}

The past couple of decades has seen a growing interest in network paradigm using low-powered devices with wireless communication capabilities, such as wireless sensor networks (WSN) and internet-of-things (IoT). WSN were first such networks, where nodes with sensing capabilities could communicate wirelessly.

WSN served as predecessor to IoT networks [12]. IoT is a computer networking architecture where "things" such as sensors and everyday objects such as smart meter and surveillance camera are connected to the internet. An IoT is a heterogeneous network, and is composed of both high-end devices such as smartphones and access points (AP), and low-end devices such as the TmoteSky sensor nodes [17]. It is projected that 10s of billions of such low-end devices, characterized as low-power low-cost devices with limited RAM and low powered microcontroller unit (MCU), will be wirelessly connected to the internet by the year 2020 [15]. The wireless channel however is inherently unreliable, which introduces errors in the received packets [22].

It has been shown that by adopting forward error correction (FEC) coding at packet level, known as erasure coding, the transmission throughput can be improved compared to retransmission based mechanism [17], [10]. Various throughput efficient erasure codes have been proposed in literature [17], [16], [14]. However, these erasure codes were designed for wireless networks with significant processing and power capabilities such as cellular, Wi-Fi, and satellite networks.

Focusing on the energy efficient operation of error recovery, this paper proposes a solution that simultaneously reduces encoding-decoding computational complexities as well as reception redundancy, so that the total energy cost of the network can be kept low. We achieve this by proposing a sparse parallel concatenated coding (SPCC) scheme, in which codes over $G F(2)$ and larger field size of $G F(32)$ are concatenated. Coding over $G F(2)$ enjoys low encoding-decoding computational complexities but introduces high reception redundancy. On the other hand, coding over $G F(32)$ offers low reception redundancy at a high encoding-decoding computational complexities.

\section{A. Related Work}

Heide et al. studied the energy cost of various wireless topologies using network coding [7]. However, in their model they only consider energy cost of transmission, reception and idle period, and do not evaluate the energy cost of encoding and decoding. 
Nistor et al. proposed an improved energy profile for coded transmission on WSN [13]. Their energy measurement technique is most closely related to our work. In their work, they consider the total energy cost due to transmission, reception of ACK, processing, idle/listen, sleeping and switching. However, in their work, they assume that the receiver is not a low-power device, and hence do not consider the energy cost of packet decoding, transmission of ACK frames and reception of data packets. Furthermore, their work does not compare the energy cost variation due to different decoding schemes, and assume that reception redundancy is zero, which may not necessarily be true.

\section{B. Main Contribution}

We show that an appropriate ratio of $G F(2)$ to that of $G F(32)$ sparse coded packets for different values of $k$ achieves low encoding-decoding computational complexities with low reception redundancy. By delimiting predefined consecutive elements of the coding vector to be always equal to zero and using table based random number minimizes the energy cost of generating random numbers, needed during encoding and decoding. With this solution, we perform testbed implementation on TelosB motes, and show that our proposed coding scheme reduces the energy cost by up to $100 \%$ compared with the next best performing coding scheme. As the energy gain increases with the packet generation size $k$, for a larger $k$, an energy cost reduction of more than $100 \%$ can be observed in devices with higher RAM.

To the best of our knowledge, this is the first work of its kind to study the total energy cost of a wireless network using erasure coding. Unlike previous results [9], [16] which adopts the big O notation for quantifying the asymptotic computational complexity of encoding and decoding, or simulation techniques, we precisely measure the exact energy consumed in millijoule $(\mathrm{mJ})$ by measuring the electric current drawn during the experiment.

\section{Organization}

The rest of the paper is organized as follow. We first present system model and problem formulation in Section II. Bibliography of related works and an overview of computational process during encoding and decoding is presented in Section III. We then present our proposed erasure coding scheme SPCC, along with analytical results of the code optimization in Section IV. We evaluate the performance of SPCC using testbed implementation in Section V. We finally conclude with the main results of our paper and future research direction in Section VI. 


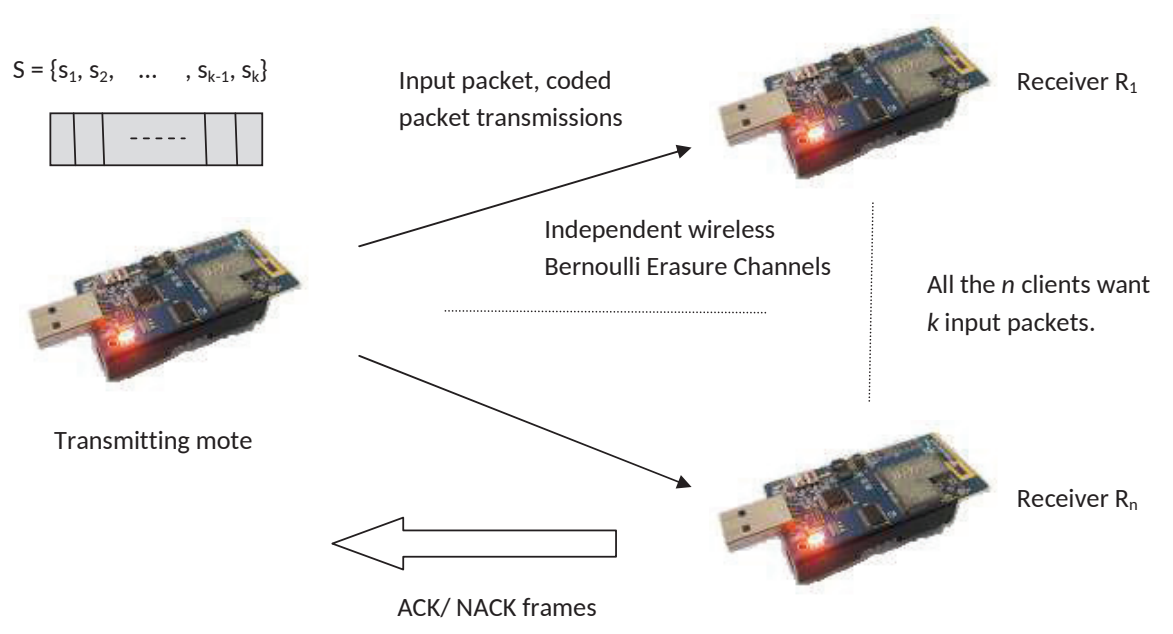

Fig. 1. Scenario of our problem. Low-powered single hop wireless unicast/ multicast network using erasure coding to improve transmission reliability.

\section{SYSTEM OVERVIEW}

Consider the scenario of $k$ input packets being requested by $n$ receivers in a single-hop star topology, as illustrated in Fig. 1. The $k$ input packets are denoted by the vector $S=\left[s_{1}, s_{2}, \ldots, s_{k}\right]$. Packet erasure at each of the receiver is assumed to be independent and identically distributed (iid), which follows the Bernoulli model with packet erasure probability of $p$.

To generate a coded packet, the transmitter first generates a coding coefficient vector $G_{j}=$ $\left[g_{1}, g_{2}, \ldots, g_{k}\right], g_{i} \in G F(q)$, which is then multiplied with the input packets and XOR added to generate coded packet $c_{j}$ given as, $c_{j}=\sum_{i=1}^{k} g_{i} \cdot s_{i}$. The term $q$ is the size of the Galois field. Zero coding vector is not used as it does not produce any useful information [21]. The probability of zero elements over field size $G F(q)$, and non-zero elements in $G_{j}$ indicates the level of sparsity and density of the coding vector respectively.

A parallel concatenation of the code is given by the generator matrix, $G=\left(G^{\prime} \mid G^{\prime \prime}\right)^{T}$, where $G^{\prime}$ is the generator matrix for code over $G F(2)$ and $G^{\prime \prime}$ is the generator matrix for code over the larger field size such as $G F(32)$. The code ratio $\varphi$ is the ratio of coded packet over $G F(2)$ received by the receiver to $k$.

A received packet is said to be linearly dependent if it can be generated by linear combination of received packets, and linearly independent otherwise. Decoding probability is the probability that a receiver has collected $k$ linearly independent packets on receiving $m=k+\delta$ packets. 
Reception redundancy $E[\delta]$ is the expected number of linearly dependent packets $\delta$ which a receiver receives before collecting $k$ linearly independent packets. The expected number of transmissions before a receiver receives $\pi$ packets is denoted by $E\left[T_{\pi}\right]$. These $\pi$ packets includes both linearly independent and dependent packets.

Once a receiver has collected $k$ linearly independent coded packets, decoding is performed as $(H \mid C)^{-1}$, where $H \in G F(q)^{m \times k}$, represents the matrix of the $m$ coding vectors $G_{j}$, and the matrix $C$ represents the matrix of received packets. Therefore, for a coding scheme with high redundancy, the size of the matrix on which the receiver needs to perform matrix inversion increases.

One efficient approach to reduce the energy consumption of sensor nodes when multicasting a set of packets over an erasure channel is to transmit coded packets rather than raw input packets. In the event of packet erasure, with coded packets, the sender can transmit missing packets to multiple receivers simultaneously which reduces the number of transmissions and energy cost. However, the use of coded packets not only introduces additional energy cost in encoding and decoding, but also the energy cost in transmitting additional $\delta$ packets due to reception redundancy. Our objective of this study are to (i) investigate the energy consumption of wireless transmissions involving packet coding over $G F(q)$, and (ii) propose parallel concatenation of packet coding scheme that can achieve overall low energy consumption.

\section{Computational Aspects of Erasure Coding}

We shall discuss the characteristics of various erasure coding presented in this section. A primer and tutorial on erasure coding techniques is presented in [16]. In random linear network coding (RLNC) each of the coding coefficient $g_{i}$ is randomly and uniformly selected from $G F(q)$. It has been shown that for RLNC over large field size $q \geq 32$, reception redundancy is near zero [21]. While for RLNC over the binary field size of $G F(2)$, a receiver needs to collect an average of $k+1.6$ packets before successful decoding [21].

The encoding and decoding computational complexities of RLNC is given as $O\left(k^{2} L\right)$ and $O\left(k^{3}+k^{2} L\right)$ respectively, assuming multiplication table is used for multiplication, where $L$ is the length of the packet. For the decoding complexity the term $k^{3}$ is the complexity of inverting the coding vector matrix using Gaussian elimination, and $k^{2} L$ is the complexity of multiplying the coded packets. An analytical model of the exact number of computation steps for Gaussian elimination is given in [4]. 
While the scale of the encoding and decoding complexities of RLNC over different field sizes is same, encoding and decoding over larger field size is more expensive computationally. In an implementation of an optimized RLNC over $G F(2)$ and RLNC over $G F(256)$ on TmoteSky sensor, it has been shown that decoding packets generated using optimized RLNC over $G F(2)$ is at least 6.5 times faster than decoding coded packet generated using RLNC over $G F(256)$ [17].

Luby-Transform (LT) code [9], [17], [16] uses sparse coding vector where log $k$ elements in the coding vector are non-zero, resulting in decoding complexity of $O(L k \log k)$. LT code is a class of fountain code due to its rateless characteristics. As LT code uses only back-substitution method for matrix inversion, and sparse coding vector, it suffers from high reception redundancy. For small values of packet generation size of $k \leq 50$ used in ultra-low power wireless network the number of redundant packets of an optimized LT code can reach as high as $40 \%$ of the value of $k$ [9].

SYNAPSE is a "hybrid" of RLNC over $G F(2)$ and an optimized LT code [17]. The objective of such SYNAPSE fountain coding scheme is to design a coding scheme with decoding complexity smaller than that of RLNC over $G F(2)$, but with relatively low reception redundancy than that of LT code. In the next two subsections we shall describe the computation required for encoding and decoding.

\section{A. Encoding}

The computational steps of encoding involve generating the coding vector, vector multiplication and XOR addition. The coding vector can be generated using encoding algorithm such as Weight-Pick [8] which makes encoding decision based on packet reception information of

all receivers. However, it is not scalable to collect feedback frames from $n$ receivers due to the shared medium of wireless channel.

Erasure coding scheme such as fountain codes [16], [17] are preferred for practical implementation where coding vectors are randomly generated. These coding vectors are generated using the pseudorandom number generator (RNG), commonly implemented using linear congruential method [5]. Instead of providing the coding vector in each packet which may significantly lengthen the packet sizes, only the seed of RNG is given in the header of each packet. This way, the receiver can regenerate the coding vector locally.

The encoding process requires vector multiplication. However, for coding over $G F(2)$, the multiplication operations are simply logical XOR operation, and hence the complex multipli- 
Fig. 2. Illustration of inactivation decoding scheme on an example matrix.

cation operations can be replaced by logical XOR operations to significantly reduces energy consumption.

The level of sparsity in the coding vector also affects the computational complexity. Generally, a higher level of sparsity reduces the needs for multiplication and the number of XOR addition operations to generate the coded packet $c_{j}$. However, a tradeoff of using high sparsity is that it increases the probability of having columns with all entries equal to zero in $H$ and hence increases reception redundancy $\delta$.

\section{B. Decoding}

The decoding operation, $(H \mid C)^{-1}$, involve matrix inversion, vector multiplication and XOR addition. Coding schemes in which coding vectors are randomly generated, the decoding step may also require additional steps of re-generating the random coding vector using the seed value for RNG [9], [17], [10]. The coding vector is seldom included in the packet header, as it incurs an overhead of $k \log _{2} q$ bits. Instead, the transmitter adds the seed value of the random number generator in the packet header for the receiver to generate the identical coding vector locally. We note here that different seed values are used to generate coding vectors for each of the coded packet.

Inactivation decoding scheme has been proposed to reduce the decoding complexity in sparse matrices. In an inactivation scheme, the decoder first performs back-substitution which reduces a submatrix of $H$ in to a reduced row echelon form. Gaussian elimination is then performed on a smaller submatrix. An illustration of inactivation decoding scheme is shown in Fig. 2.

\section{Sparse Parallel Concatenation Code (SPCC)}

In this section, we shall explain our proposed method of erasure code, SPCC. We recognize that coding over $G F(2)$ enjoys low encoding-decoding computation cost, however it incurs high 
TABLE I

Summary of VARIOUS ERASURE CODING SCHEMES. THE VAlues of $E[\delta]$ FOR $G F(2)$ AND $G F(32)$ IS VALID FOR $k \geq 5$ AND $k \geq 2$ RESPECTIVELY.

\begin{tabular}{|c|c|c|c|c|c|}
\hline & RLNC $G F(2)[21]$ & RLNC $G F(32)[21]$ & LT code [9] & SYNAPSE [17] & Proposed (SPCC) \\
\hline Expected Redundancy & $E[\delta]=1.6$ & $E[\delta]=0.033$ & High & $E[\delta] \approx 2.5$ & $E[\delta] \approx 0.1$ \\
Encoding Decision & RNG & RNG & RNG & RNG & Table-lookup \\
Density & 0.5 & 0.97 & $\frac{\log k}{k}$ & Low-Medium & Low \\
Encoding & XOR & XOR, MULT & XOR & XOR & XOR, MULT \\
Decoding & GE, XOR & GE, XOR, MULT & BS, XOR & GE, XOR & ID, XOR, MULT \\
No. of Multiplications & - & High & - & - & Very low \\
\hline \multicolumn{7}{l|}{ Key: MULT- multiplication table, GE- Gaussian elimination, BS- Back substitution, ID- Inactivation decoding. } \\
\hline
\end{tabular}

reception redundancy. On the other hand, coding over $G F(32)$ requires relatively higher encodingdecoding computation cost, but it offers low reception redundancy. Our proposed scheme mixes $G F(2)$ and $G F(32)$ by parallel concatenating the two to take advantage of the lower encodingdecoding computation cost from $G F(2)$ code and lower reception redundancy from $G F(32)$.

To ensure that SPCC can capture both the low computational cost and low reception redundancy features, it is necessary to (i) select appropriate sparsity settings for $G F(2)$ and $G F(32)$, and (ii) balance the use of $G F(2)$ and $G F(32)$ during concatenation for low overall energy cost. In the following, we first study the sparsity of $G F(2)$ and $G F(32)$ for appropriate settings, and then derive a closed form expression which models the expected number of redundancy for a given code ratio. The analytical results permit us to further calculate the overall energy cost and select optimal code ratio.

While $G F(q)$ with $q>32$ may be used to minimize reception redundancy, a higher $q$ setting drastically increases the demands on memory usage for the multiplication table given as $q^{2} \log _{2} q$ bits. To maintain practicability, our design uses $G F(32)$ which is appropriate for ultra-low powered network devices. A summary of the main characteristics of the existing and proposed erasure coding scheme is given in Table I.

\section{A. Sparsity Setting}

In a sparse RLNC the probability of selecting zero coding coefficient is given by $p_{s, q}$, and each of the non-zero elements are selected with a probability of $\frac{1-p_{s, q}}{q-1}$. The exact closed form 
expression for singularity probability analysis of sparse RLNC remains an open problem even with the most recent works [1], [20].

We shall now establish the optimal settings of sparsity for both $G F(2)$ and $G F(32)$ given a particular $k$. These optimal settings shall be used for our design. We first quantify the energy costs of encoding and decoding, as well as redundant transmissions and receptions for various sparsity $p_{s, q}$ and $k$, then illustrate the tradeoff between encoding-decoding and reception redundancy for sparsity optimization.

Let $C_{L}, C_{T}$ and $C_{R}$ be the energy cost of table lookup (for multiplication and XOR addition), transmission and reception for a particular sensor node respectively. In this paper, we consider TelsoB devices. Our experimental measure on TelosB gives $C_{L}=33.51 \mathrm{~nJ}, C_{T}=409.4 \mu \mathrm{J}$ and $C_{R}=412.22 \mu \mathrm{J}$. Recall that $m=k+E[\delta]$ is the number of coded packets involved in encoding and decoding process for $k$ input packets. The quantity $m$ can be computed based on the work presented in [1] for differen values of sparsity $p_{s, q}$. We further assume that the size of data packets is constant with length of $L$ bits.

To perform encoding, an $L$-bit packet is treated as a vector over the field size $G F(q)$ by forming symbols of $\log _{2} q$ bits [18]. Based on this, the total energy cost of encoding over $G F(q)$ denoted by $\phi_{E}(q)$ is

$$
\begin{aligned}
& \phi_{E}(2)=k\left(1-p_{s, 2}\right) \times m \times L \times C_{L} \\
& \phi_{E}(32)=k\left(1-p_{s, 32}\right) \times m \times L \times C_{L} \times\left(1+\frac{1}{\log _{2} 32}\right)
\end{aligned}
$$

where $k\left(1-p_{s, q}\right)$ is the average number of input packets used to generate a coded packet. With $L$-bit packets, the number of XOR addition is $L$, and the number of multiplications over $G F(32)$ is $\frac{L}{\log _{2} 32}$. Note that encoding over $G F(2)$ does not involve in additional multiplication operations.

To determine the energy cost of decoding we adopt the model proposed in [4] to compute the energy cost of Gaussian elimination. Based on the model presented in [4] for a non-sparse matrix the total number of multiplications and additions $S_{T}$ required to invert a $k \times(k+1)$ matrix $(H \mid C)$, while neglecting $C$ is given as

$$
S_{T}=\frac{2 k^{3}}{3}-\frac{k^{2}}{2}+\frac{5 k}{6} .
$$

We validate the result of our simulator, used to plot Figure 4, by determining the number of computation steps for matrix inversion on a dense $k \times k G F(32)$ matrix and then comparing its result with $S_{T}$ in Figure 3. The correctness of our simulator to correctly determine the expected 


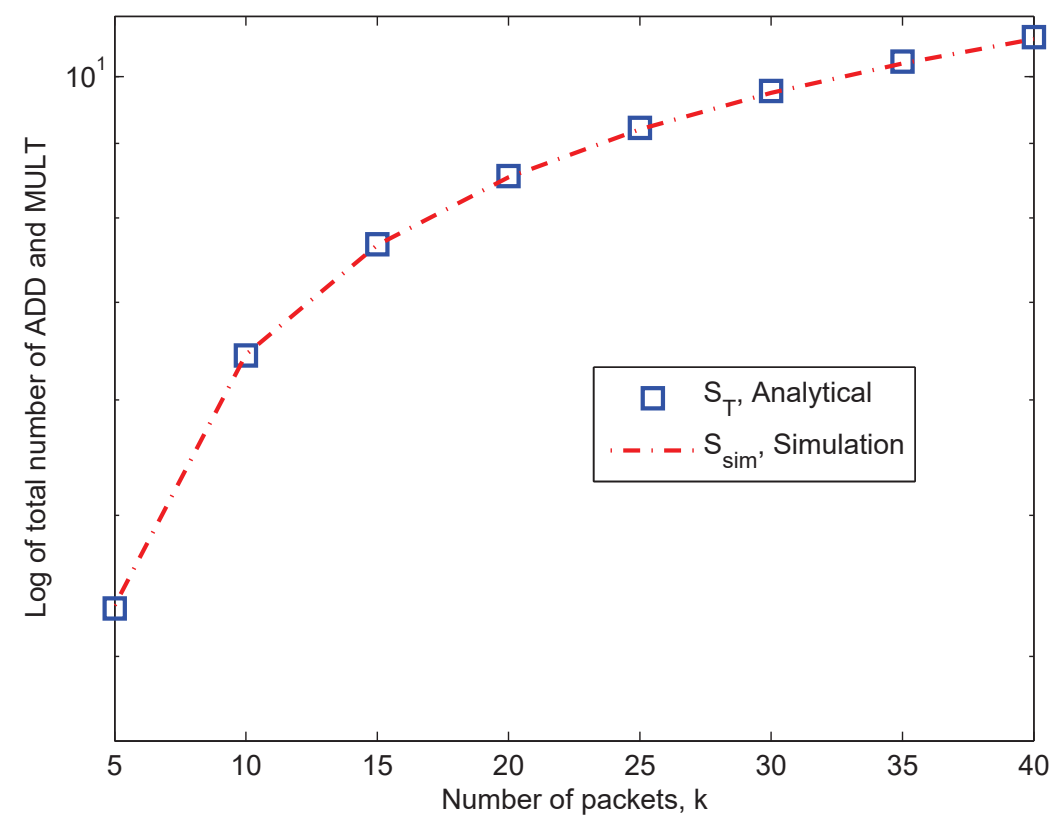

Fig. 3. The total number of ADD and MULT to invert a $k \times k$ dense matrix. The value of $S_{T}$ is negligibly higher than $S_{\text {sim }}$ due to the presence of 0 and 1 elements in a $k \times k G F(32)$ matrix which leads a small reduction in the number of additions and multiplications for $S_{\text {sim }}$.

rank of the matrix $H$ before successful decoding has been verified with the analytical model [1] as shown in Figure 4.

We use the matrix inversion simulator to determine the expected number of additions and multiplication $S_{\text {sim }}$ for $m \times(k+1)$ matrix $(H \mid C)$ with $H$ having sparsity of $p_{s, q}$. As elements of matrix $C$ corresponds to coded packets, the number of multiplications to multiply an element over the field size $G F(32)$ is given by $\frac{L}{\log _{2} 32}$, and the number of such multiplications is included in the value of $S_{\text {sim }}$.

Finally the energy cost of transmission and reception of redundancy packets by the $n$ receivers, $\phi_{T R}(q)$, is simply

$$
\phi_{T R}(q)=E[\delta]\left(C_{T}+n C_{R}\right)
$$

With the above results, given a particular number of input packets $k$ to transmit to $n$ receivers, the additional energy cost for RLNC over $G F(q)$ denoted as $\phi_{T o t}(q)$ is

$$
\phi_{T o t}(q)=\phi_{E}(q)+n \phi_{D}(q)+\phi_{T R}(q) .
$$


where $\phi_{D}(q)$ is the decoding energy cost, which is the sum of energy costs of multiplications and additions, that is $\phi_{D}(q)=S_{s i m} C_{L}$. Given that sparsity setting is the only input to the search, we can find the minimum $\phi_{T o t}(q)$ by exhaustively searching the sparsity.

We observe that the energy cost is dominated by $\phi_{T R}(q)$ which is mainly influenced by $E[\delta]$. In Fig. 5, we plot the $k+E[\delta]$ versus sparsity setting for $G F(2)$ and $G F(32)$. As can be seen, $E[\delta]$ monotonically increases as sparsity increases. When sparsity setting is high, $\phi_{T o t}(q)$ is high due to high $\phi_{T R}(q)$. As sparsity decreases, $E[\delta]$ drops, so is $\phi_{T R}(q)$, and at the same time $\phi_{E}(q)+n \phi_{D}(q)$ slowly increases. When sparsity decreases to the region when $E[\delta]$ becomes relatively flat, further decrease in sparsity introduces increase in $\phi_{E}(q)+n \phi_{D}(q)$. We illustrate this tradeoff in Fig. 5.

\section{B. Reception Redundancy}

Our proposed parallel code concatenation mixes $G F(2)$ and $G F(32)$ encoding to produce coded packets. Knowing the number of input packets $k$, it is necessary to decide how many coded packets to be encoded using $G F(2)$ and how many using $G F(32)$. This choice affected the energy cost since using excessive $G F(2)$ introduces high reception redundancy, and using excessive $G F(32)$ introduces high computation in encoding-decoding processes. Using the sparsity settings developed in the previous subsection, we further derive the overall reception redundancy for our proposed SPCC, $E\left[R_{\varphi, k}\right]-k$, for different code ratio $\varphi$. The overall reception redundancy is needed to compute the energy cost of encoding-decoding processes as well as the energy involved in additional transmissions and receptions.

Let $\varphi$ be the code ratio defined as the number of $G F(2)$ coded packets to the number of input packets $k$. Our objective is to find the optimal code ratio setting such that the overall energy cost of the network is minimal. One key factor affecting the energy cost is the reception redundancy, thus we first derive the reception redundancy for various code ratios. The sparsity settings for $G F(2)$ and $G F(32)$ are based on the results developed in the previous subsection. We further define $q_{1}$ and $q_{2}$ to be the field sizes of coding schemes over $G F(2)$ and $G F(32)$ respectively. The term $x_{1}$ and $x_{2}$ denotes the numbers of linearly dependent coded packets over $G F(2)$ and $G F(32)$ respectively.

Let $\ell$ be the number of coded packets over $G F(2)$ received by a receiver. We first note that the probability that the first received packet is independent is equal to one. The probability that the second received packet is linearly dependent is given as $\frac{q_{1}-1}{q_{1}^{k}-1}$. We use minus one in the 


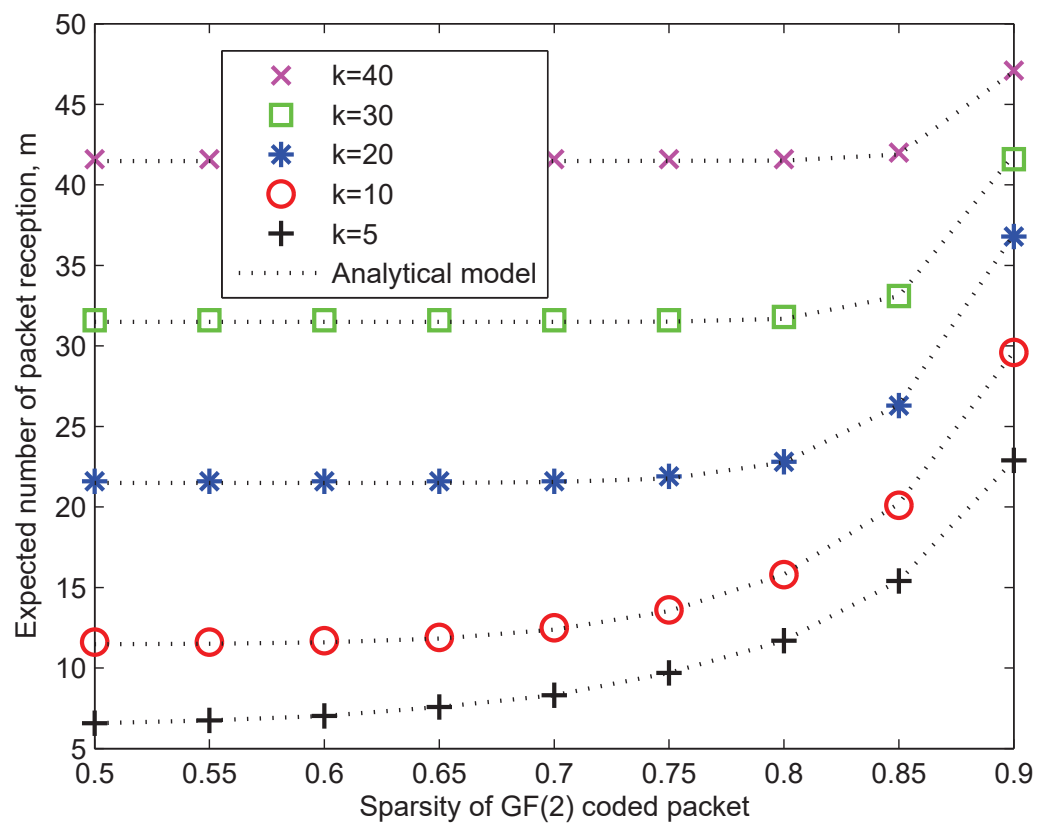

(a) Sparse RLNC over $G F(2)$

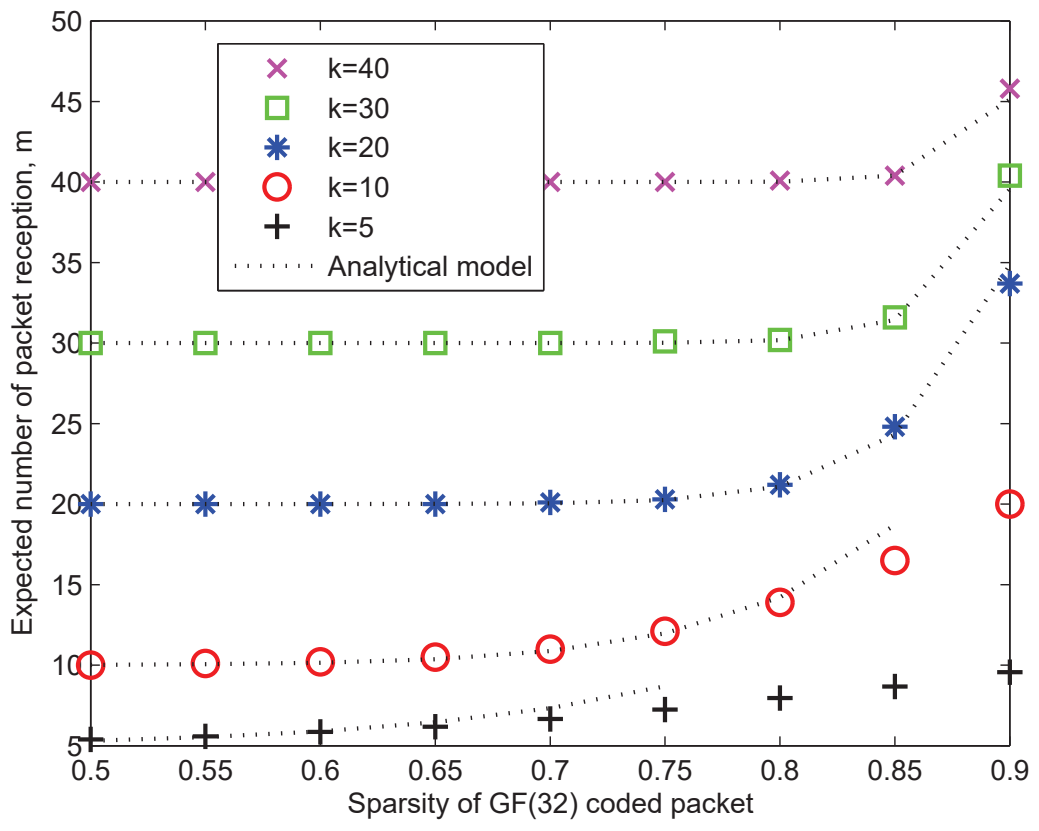

(b) Sparse RLNC over $G F(32)$

Fig. 4. The expected number of packets $m=k+E[\delta]$ a receiver need to receive with different sparsity using sparse RLNC over (a) $G F(2)$, and (b) $G F(32)$, with one example threshold point highlighted in each subfigure. For $G F(32)$ when $k$ is small and $p_{s, 32}$ is high, the mean square error (MSE) between the analytical model and Monte Carlo simulation is high [1], hence the analytical result in this region is excluded from the graph. 


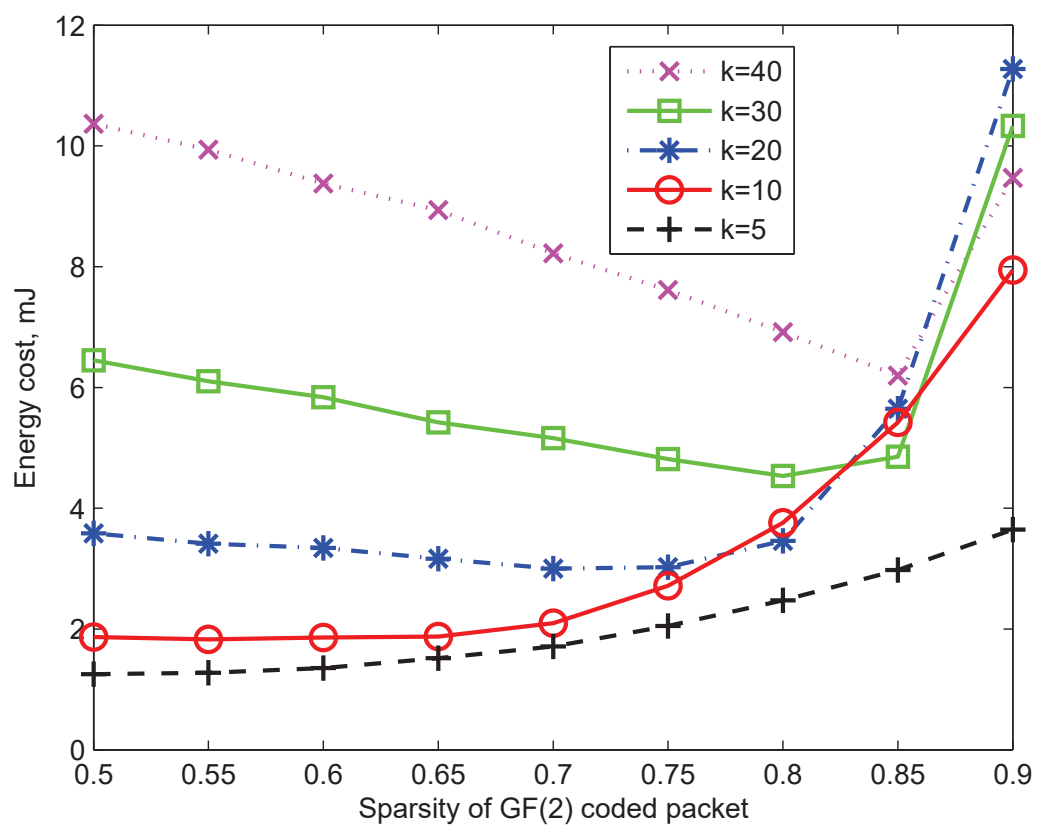

(a) Sparse RLNC over $G F(2)$

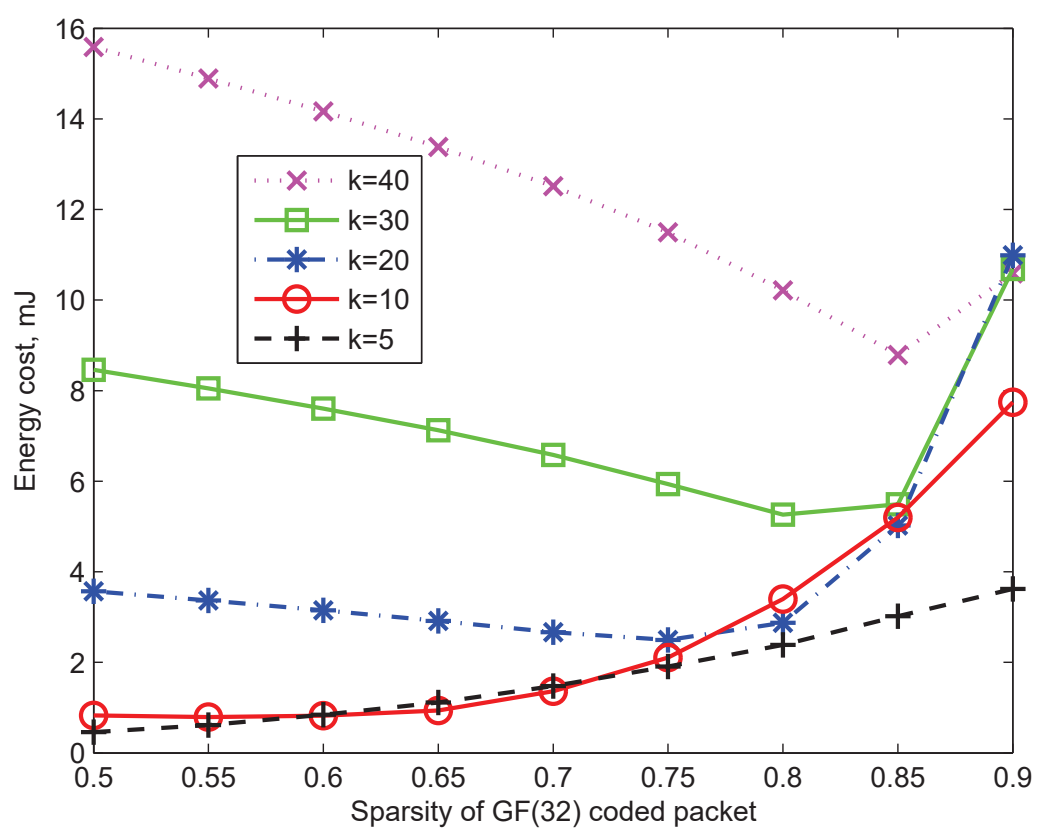

(b) Sparse RLNC over $G F(32)$

Fig. 5. The total energy $\operatorname{cost} \phi_{\text {Tot }}(q)$ for the case of a single receiver $(n=1)$ with different sparsity $p_{s, q}$ using sparse RLNC over (a) $G F(2)$, and (b) $G F(32)$. 
numerator and denominator as we exclude the zero coding vector. The term $q_{1}^{k}$ is the cardinality of the span of coding vector, and $q_{1}-1$ is the cardinality of the event that the second received packet is a multiple of the first received packet. Extending the above result, the probability that after receiving $i-1$ linearly independent packets, the $i^{\text {th }}$ received coded packet over $G F(2)$ is linearly dependent is given by,

$$
P\left(D_{q_{1}}^{i}\right)=\frac{q_{1}^{i-1}-1}{q_{1}^{k}-1},
$$

where $q_{1}^{i-1}-1$ is the cardinality of the set of linear combinations of all the $i-1$ received packets. Thus the probability that the $i^{\text {th }}$ received packet is linearly independent after the receiver has received $i-1$ linearly independent packets is simply,

$$
P\left(I_{q_{1}}^{i}\right)=1-P\left(D_{q_{1}}^{i}\right)
$$

Now we turn our focus on reception redundancy on coded packet over $G F(32)$. Similarly to the above approach, the probability that after receiving $j-1$ linearly independent packets over $G F(32)$, the $j^{t h}$ packet is linearly dependent is given by,

$$
P\left(D_{q_{2}}^{j, x_{1}}\right)=\frac{q_{2}^{\ell-x_{1}+j-1}-1}{q_{2}^{k}-1} .
$$

The probability that the $j^{\text {th }}$ received coded packet is linearly independent conditional that the receiver has received $i-1$ linearly independent packet is given by,

$$
P\left(I_{q_{2}}^{j, x_{1}}\right)=1-P\left(D_{q_{2}}^{j, x_{1}}\right)
$$

Based on the above results, we now derive the probability mass function (pmf) of successful decoding probability after a receiver has received $k+\delta$ packets. It is the probability that a full rank matrix $H$ has been formed after receiving $x_{1}$ coded packets over $G F(2)$ and $x_{2}$ coded packets over $G F(32)$.

Let $P\left(H_{k+\delta}\right)$ denote the probability that a full $k$ rank matrix is formed after receiving $k+\delta$ coded packets. We recognize that the reception redundant packets $\delta$ can be due to linearly dependent coded packets over $G F(2)$ or $G F(32)$, and thus $\delta=x_{1}+x_{2}$. The quantity $P\left(H_{k+\delta}\right)$ is the sum of all possible cases for $x_{1}$ and $x_{2}$ where $\delta=x_{1}+x_{2}$. For each case of $x_{1}$ and $x_{2}$, the receiver needs $\ell-x_{1}$ of $G F(2)$ coded linearly independent packets and $k-\ell-x_{2}$ of $G F(32)$ coded linearly independent packets to form a full rank. At the same time, the receiver also receives $x_{1}$ redundant packets coded over $G F(2)$ and $x_{2}$ redundant packets coded over $G F(32)$. Using 
TABLE II

COMPARISON OF EXPECTED NUMBER OF RECEPTIONS $E\left[R_{\varphi, k}\right]$, USING ANALYTICAL MODEL FOR CONCATENATED CODE, AND SIMULATION MODEL FOR SPCC, FOR DIFFERENT CODE RATIO.

\begin{tabular}{|c|cc|cc|cc|}
\hline & \multicolumn{2}{|c|}{$k=10$} & \multicolumn{2}{c|}{$k=20$} & \multicolumn{2}{c|}{$k=30$} \\
\hline Ratio & $E\left[R_{\varphi, k}\right]$ & Simulation & $E\left[R_{\varphi, k}\right]$ & Simulation & $E\left[R_{\varphi, k}\right]$ & Simulation \\
\hline 0.2 & 10.0342 & 10.0898 & 20.0333 & 20.0839 & 30.0333 & 30.0496 \\
\hline 0.3 & 10.037 & 10.1063 & 20.0333 & 20.0862 & 30.0333 & 30.0590 \\
\hline 0.4 & 10.044 & 10.1114 & 20.0335 & 20.0884 & 30.0333 & 30.0587 \\
\hline 0.5 & 10.0586 & 10.1286 & 20.0342 & 20.0933 & 30.0333 & 30.0550 \\
\hline 0.6 & 10.0885 & 10.1609 & 20.0372 & 20.0905 & 30.0335 & 30.0530 \\
\hline 0.7 & 10.1483 & 10.2375 & 20.0488 & 20.0961 & 30.0352 & 30.0599 \\
\hline 0.8 & 10.2653 & 10.3567 & 20.0951 & 20.1574 & 30.0488 & 30.0752 \\
\hline 0.9 & 10.4855 & 10.5752 & 20.2731 & 20.3484 & 30.1557 & 30.1911 \\
\hline
\end{tabular}

the results given in Equations (1)-(4), iterating all cases where $x_{1}+x_{2}=\delta$, we establish the following

$$
\begin{gathered}
P\left(H_{k+\delta}\right)=\sum_{\substack{\forall x_{a}: x_{1}+x_{2}=\delta, 0 \leq x_{a} \leq \delta}}\left\{\prod_{i=2}^{\ell-x_{1}} P\left(I_{q_{1}}^{i}\right) \cdot \prod_{j=1}^{k-\ell+x_{1}} P\left(I_{q_{2}}^{j, x_{1}}\right) \cdot\right. \\
\sum_{\substack{\forall d_{t}: d_{1} \leq \ldots \leq d_{x_{1}}, 2 \leq d_{t} \leq \ell-x_{1}+1}}\left(\prod_{t=1}^{x_{1}} P\left(D_{q_{1}}^{d_{t}}\right)\right) . \\
\left.\sum_{\substack{\forall d_{t}: d_{1} \leq \ldots \leq d_{x_{2}}, 1 \leq d_{t} \leq k-\ell}}\left(\prod_{t=1}^{x_{2}} P\left(D_{q_{2}}^{d_{t}, x_{1}}\right)\right)\right\} .
\end{gathered}
$$

Given the above expression, the expected number of coded packets $E\left[R_{\varphi, k}\right]$ a receiver needs to receive for a given value of $\ell$ based on sparsity of $\varphi$ before collecting $k$ linearly independent coded packet can be given by,

$$
E\left[R_{\varphi, k}\right]=k+\sum_{\delta=0}^{\infty} \delta \cdot P\left(H_{k+\delta}\right) .
$$

The expected number of transmissions before a receiver has collected $\ell$ coded packets over $G F(2)$ can be derived using the pmf of negative binomial distribution as,

$$
E\left[T_{\ell}\right]=\sum_{\tau=\ell}^{\infty} \tau \cdot\left(\begin{array}{l}
\tau-1 \\
\ell-1
\end{array}\right)(1-p)^{\ell} p^{\tau-\ell} .
$$


The result given in Equation (6) can be used by the transmitter to estimate when to switch transmitting coded packets over $G F(32)$. When an estimation of packet erasure probability is unknown to the transmitter, an arbitrary receiver can be selected to transmit control frames to inform the transmitter that it has collected $\ell$ packets. Channel statistic is commonly used to estimate packet erasure probability [3].

The expected number of transmissions before a receiver has collected $k$ linearly independent packets when using the SPCC scheme is given by,

$$
\begin{aligned}
E\left[T_{k}\right] & =\sum_{\delta=0}^{\infty} P\left(H_{k+\delta}^{\ell}\right) \cdot \\
& \left(\sum_{\tau=k+\delta}^{\infty} \tau \cdot\left(\begin{array}{c}
\tau-1 \\
k+\delta-1
\end{array}\right)(1-p)^{k+\delta} p^{\tau-k-\delta}\right) .
\end{aligned}
$$

The term in the bracket represents negative binomial distribution, i.e. the probability that a receiver has successfully received $k+\delta$ packets after exactly $\tau$ transmissions. The term $P\left(H_{k+\delta}^{\ell}\right)$ represents the decoding probability after a receiver has received $k+\delta$ packets, of which $\ell$ packets are coded over $G F(2)$ and the remaining over $G F(32)$. We validate the correctness of our derivation using simulation. The numerical and simulation results are compared in Table II showing good agreement with each other. We also notice that the reception redundancy remains flat while code ratio is low, but begins to increase obviously when code ratio researches a certain value. This gives us opportunity to pick an adequate code ratio that incurs low reception redundancy as competitive as sparse RLNC yet utilizes more coding over $G F(2)$ for low computational cost in encoding-decoding. We shall investigate the code ratio optimization in the next subsection.

\section{Code Ratio Setting}

The code ratio setting in SPCC influences the energy usage in batch transmissions. SPCC behaves like sparse RLNC over $G F(32)$ when code ratio is low and sparse RLNC over $G F(2)$ when code ratio is high. Ideally, we want the code ratio to be large so that the computational cost can be reduced. However, a larger code ratio also introduces increased reception redundancy. Based on our study in the previous subsection, we found that the reception redundancy remains relatively flat for some small code ratio settings and only increases obviously when the code ratio setting reaches a certain value. In this subsection, we shall find an operating point that minimizes the overall energy cost. We derive an analytical model to optimize the code ratio for different values of $k$. 
For a given value of $k$ the energy cost of encoding $\xi_{E}(\ell)$ for different values of $\ell$ is given as,

$$
\xi_{E}(\ell)=k\left(1-p_{s, q}\right) \times L \times C_{L} \times\left(\ell+(m-\ell)\left(1+\frac{1}{\log _{2} 32}\right)\right)
$$

as $\ell$ coded packets are generated by only XOR addition, and $m-\ell$ coded packets are generated by multiplication and XOR addition. Besides, as presented in Fig. 5, the optimal value of $p_{s, 2}$ coincide with that of $p_{s, 32}$, we simply use a single term $p_{s, q}$ in the above calculation to determine $\xi_{E}(\ell)$.

For different values of $\ell$ we use our matrix inversion simulator discussed in Section IV-A to obtain $S_{\text {sim }}$, the value of total number of multiplications and XOR additions. The use of simulation gives more precise result than the analytical one since the analytical result assumes non-sparse matrix. The value of $S_{s i m}, n$ and $C_{L}$ can then be used to determine the energy cost of decoding $\xi_{D}(\ell)$. The energy cost of transmitting and receiving redundant packets is given as

$$
\xi_{T R}(\ell)=\left(E\left[R_{\varphi, k}\right]-k\right) \times\left(C_{T}+n C_{R}\right)
$$

The total energy cost $\xi_{T o t}(\ell)$ is given by the summation of $\xi_{E}(\ell), \xi_{D}(\ell)$ and $\xi_{T R}(\ell)$. A plot of $\xi_{\text {Tot }}(\ell)$ against code ratio $\varphi$ is plotted in Fig. 6. The result of the graph shows that for small values of $k$, most of the coded packets should be generated over $G F(32)$. For example, for $5 \leq k \leq 10$ a code ratio of in the interval of $[0,0.4]$ should be used. However for larger values of $k$, most packets should be generated over $G F(2)$.

The result given in Fig. 6 can be explained as follow. Sparse RLNC over $G F(2)$ have redundancy of approximately 1.6 packets. For small values of $k$, the redundancy ratio $\frac{1.6}{k}$ will be high which can contribute to significant additional energy cost. This can be mitigated by using sparse RLNC over $G F(32)$. While such code over $G F(32)$ have higher encoding and decoding cost due to multiplication, due to small size of the $m \times k$ matrix $H$ for small values of $k$ such additional energy cost is relatively modest. As the value of $k$ increases, the ratio $\frac{1.6}{k}$ decreases, and the effect of redundancy due to $G F(2)$ towards the total energy cost gradually becomes irrelevant, in which case the advantage of coding over $G F(32)$ to minimize redundancy gradually diminishes, and the energy cost of multiplications to invert a matrix for larger $k$ increases by cubic factor. This therefore makes coding mostly over sparse $G F(2)$ code advantageous for larger values of $k$. 


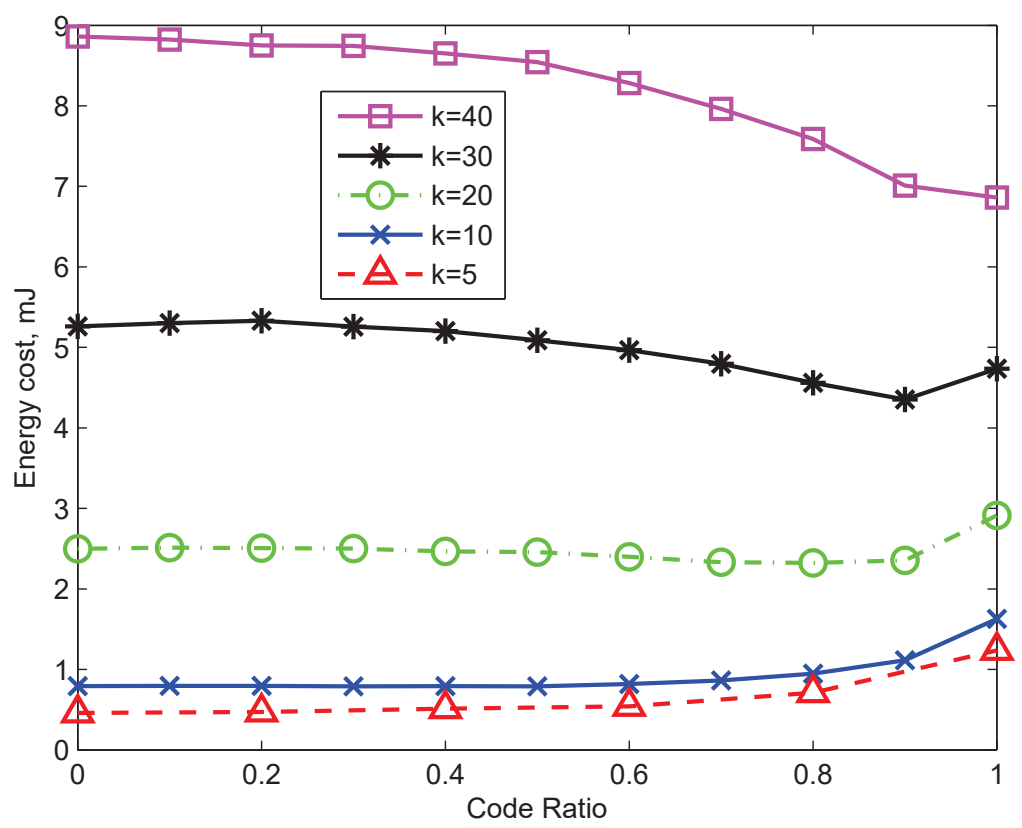

Fig. 6. The total energy cost $\xi_{T o t}(\ell)$ for different code ratio $\varphi$

\section{Coding Vector}

In the earlier section, we have described the current approach to reduce packet sizes by avoiding explicitly providing coding vector in the header. While the approach reduces packet sizes, it demands the receiver to compute coding vectors using complex RNG.

To further minimize the energy cost of generating the coding vector using RNG, we use an approach where $\eta$ consecutive coding coefficient are assigned values uniformly and randomly selected from $G F(q)$, and the remaining $k-\eta$ coefficients are assigned zero values. This requires us to compute the starting position, which we call pivot, from where onwards $\eta$ coding coefficients will be randomly selected from $G F(q)$. The coding coefficient at pivot position will always be equal to one for $G F(2)$, and uniformly and randomly selected from the range $[1,31]$ for $G F(32)$.

In fact to maintain the same statistical properties of the coding coefficients, the pivot vector for $G F(q)$ should be uniformly and randomly selected from $q$. However, this will incur the overhead of searching for the first non-zero entry while performing Gaussian elimination should the pivot and coefficients following the pivot be equal to zero. By fixing the pivot position to 
be equal to a non-zero coefficient, the value of $\eta$ can be obtained as,

$$
\eta \cdot \frac{q-1}{q}+1=k\left(1-p_{s}\right), \text { hence } \eta=\frac{q\left(k\left(1-p_{s}\right)-1\right)}{q-1},
$$

where $p_{s}$ is the optimal sparsity setting. After obtaining the value of $\eta$, we round it up to the nearest integer so that the reception redundancy is lower than the target.

Randomly generated values in the range $[1, k]$ are stored in one of the table to find the value of pivot. Considering a packet generation size of 50 due to limited RAM of low power devices, being transmitted over a wireless channel with $50 \%$ erasure probability, the expected length of such table will be 100 entries. For multicast transmission with higher erasure probability, such length will not exceed few hundreds of entries.

In two other tables we store random numbers uniformly and randomly selected from $G F(2)$ and $G F(32)$. We use sliding window on the table entries to determine the coding coefficients for the $\eta$ coding coefficient once the pivot has been selected as illustrated in Fig. 7. Where the sliding window moves one position on the left once a vector has been selected.

When the difference between $k$ and the value of pivot is less than $\eta$, then after reaching the tail of the vector, entries starting at the head of the vector are filled up. Both the transmitter and receivers will have the same tables, and therefore it is sufficient for the transmitter to include the starting point of the sliding window in the header of the packet, which the receiver can use to determine the pivot position, and the remaining $\eta$ coding coefficients. Our method essentially trades storage for lower computation operation to reduce power consumption.

\section{Testbed Evaluation}

We verify the effectiveness of SPCC using testbed implementation. We use the Advanticsys CM5000 TelosB mote running the TinyOS, with MSP430 MCU and CC2420 radio frequency (RF) Chip. The MSP430 is a low-power MCU, with 10KB of RAM. The CC2420 is a $2.4 \mathrm{GHz}$ IEEE 802.15.4 compliant RF chip, with an effective data rate of $250 \mathrm{Kbps}$.

The effective time taken for the RF transceiver to transmit a packet of fixed packet size also needs to take in to consideration the time to perform processes such as the carrier sense multiple access collision avoidance (CSMA/CA), and data transfer time between MCU and RF chip on the serial peripheral interface (SPI) bus.

In the IEEE 802.15.4-2003 standard, two CSMA/CA protocols have been proposed, unslotted CSMA/CA and the optional slotted CSMA/CA. TelosB uses the unslotted CSMA/CA, in which 


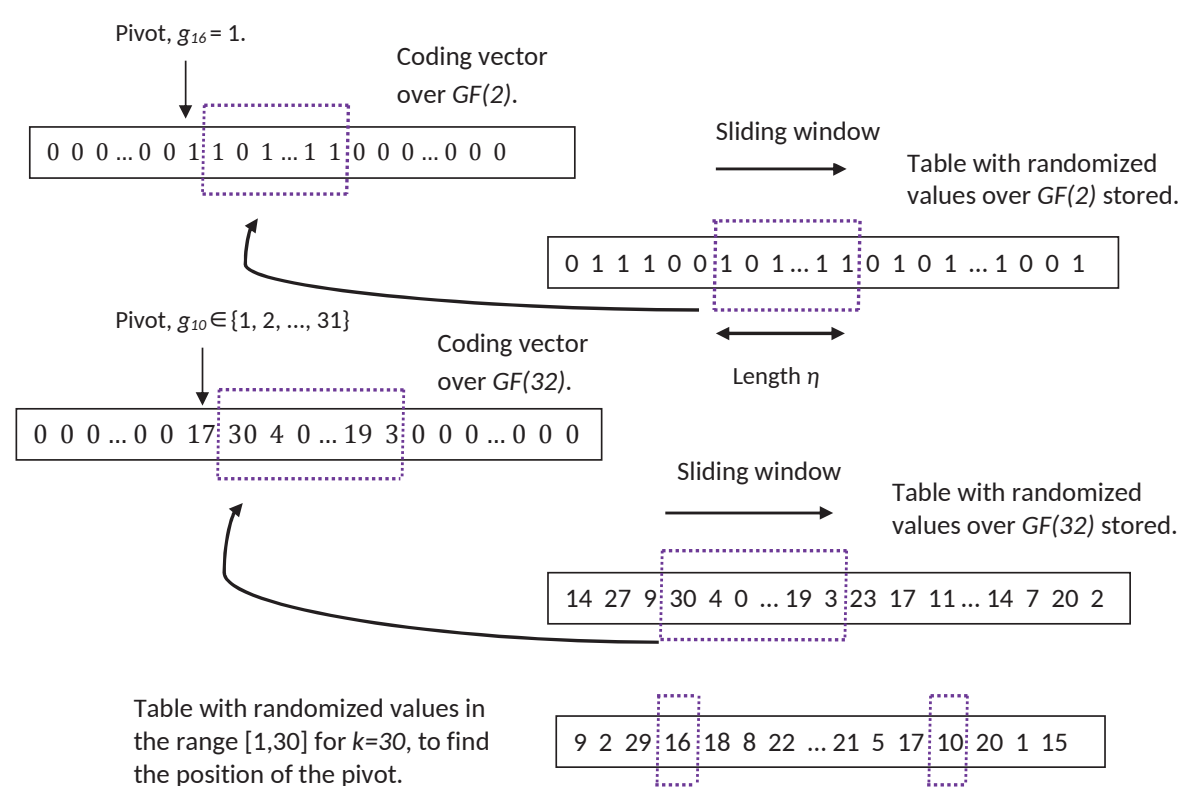

Fig. 7. Illustration of coding vector generation tables.

the mote randomly waits for a duration of $\left[0,2^{B E}-1\right]$ slots of aUnitBackoffPeriod before performing the clear channel assessment (CCA) to determine whether the channel is free. Where $B E$ is the back-off exponent, with a default value of 3 , and a maximum value of 5 , and the duration of aUnitBackoffPeriod slot is $320 \mu \mathrm{s}$. The time duration to perform CCA is $128 \mu \mathrm{s}$. In the absence of any interference, the expected time for CSMA/CA is $1.248 \mathrm{~ms}$. According to testbed evaluation of packet transmission by 802.15.4 Zigbee mote without any interfering transmission, the expected time for CSMA/CA was found to be $1.6 \mathrm{~ms}$ [2].

The data transfer rate on the SPI is approximately given as $0.31 m s+(L+11) 46 \mu s$ [19], where $L$ is the length of the data packet. The time taken to transmit $L$ bytes is given as $0.99 m s+\frac{8 \times L}{250}$ [2]. The turnaround time to switch between transmission and reception mode (TX/RX) by the RF chip is $192 \mu \mathrm{s}$.

We practically evaluated the inter packet arrival time for $L=20$ bytes without encodingdecoding operations (the experiments in [22] similarly used packet size of 18 bytes for 802.15.4 Micaz motes), and found the average time to be approximately equal to $5.8 \mathrm{~ms}$ (based on our measurement this corresponds to an average energy consumption of $409.4 \mu \mathrm{J})$. Our result closely matches with the inter packet arrival time of 5.5ms reported in [6] for Tmote Sky mote using CC2420. The slightly higher time in our result can be explained due to the presence of Wi-Fi 


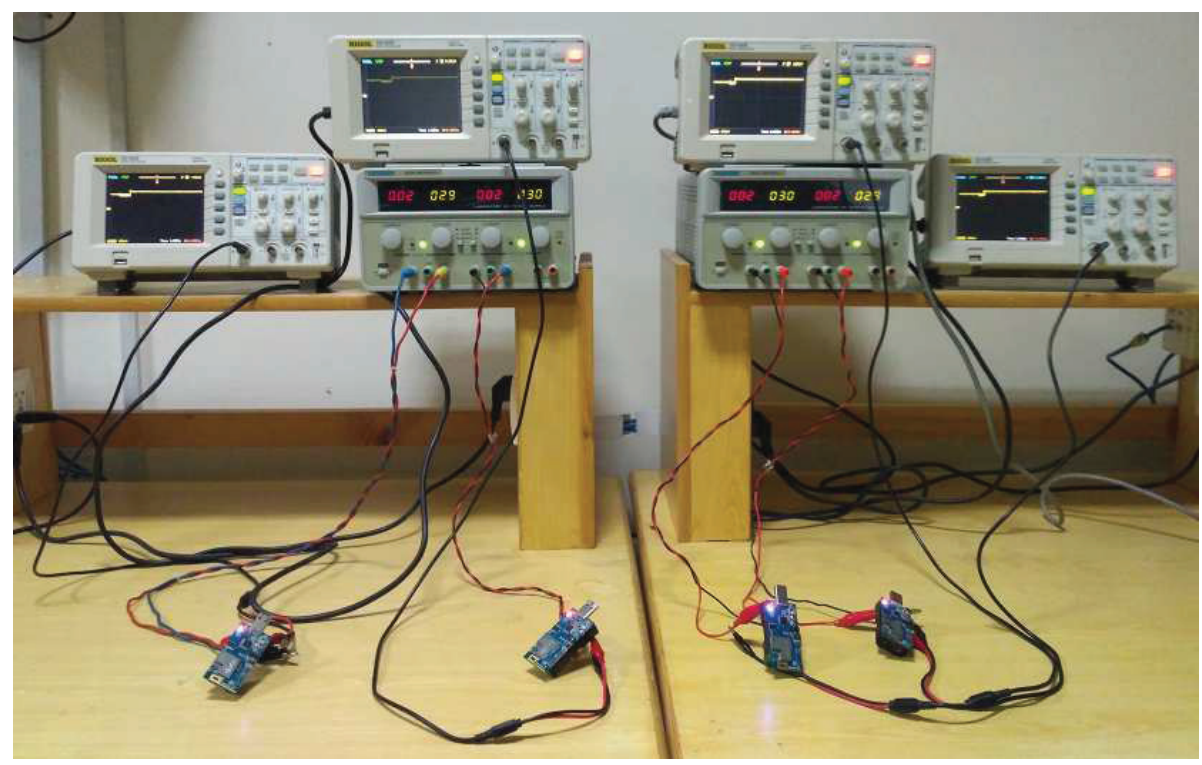

Fig. 8. Experimental setup of our testbed implementation, with four receiving TelosB motes. The transmitting TelosB mote (not shown) is placed approximately 3 meters away. The average packet loss probability observed during the experiment was $p=0.1$.

transmission operating in the $2.4 \mathrm{GHz}$ band during experimentation, which can increase the expected time of CSMA/CA protocol.

As the energy used by the RF chip will fluctuate [2] when performing different operations such as CSMA/CA, TX/RX, packet transmission, and packet reception we use digital storage oscilloscope (DSO) based circuit to measure the energy consumed by the mote during the $5.8 \mathrm{~ms}$ time interval for transmission. Similarly, the current drawn by the MCU will fluctuate as it performs various operations such as sending and receiving data to CC2420 processor, XOR addition, as well as multiplication table lookup during encoding and decoding.

\section{A. Results}

We set up experimental testbeds with one and four receiving TelosB motes, and one transmitting TelosB mote as illustrated in Fig. 8 for the case of four receivers. To evaluate the energy cost, we measure the current of the receivers since the receiver receives the first data packet, until it has decoded all the $k$ packets, and the current of the transmitter since it transmits its first packet until it has received an ACK from all the four receivers. We then obtain the energy cost (in Joules) by multiplying the voltage supplied to the mote, the average current measured 


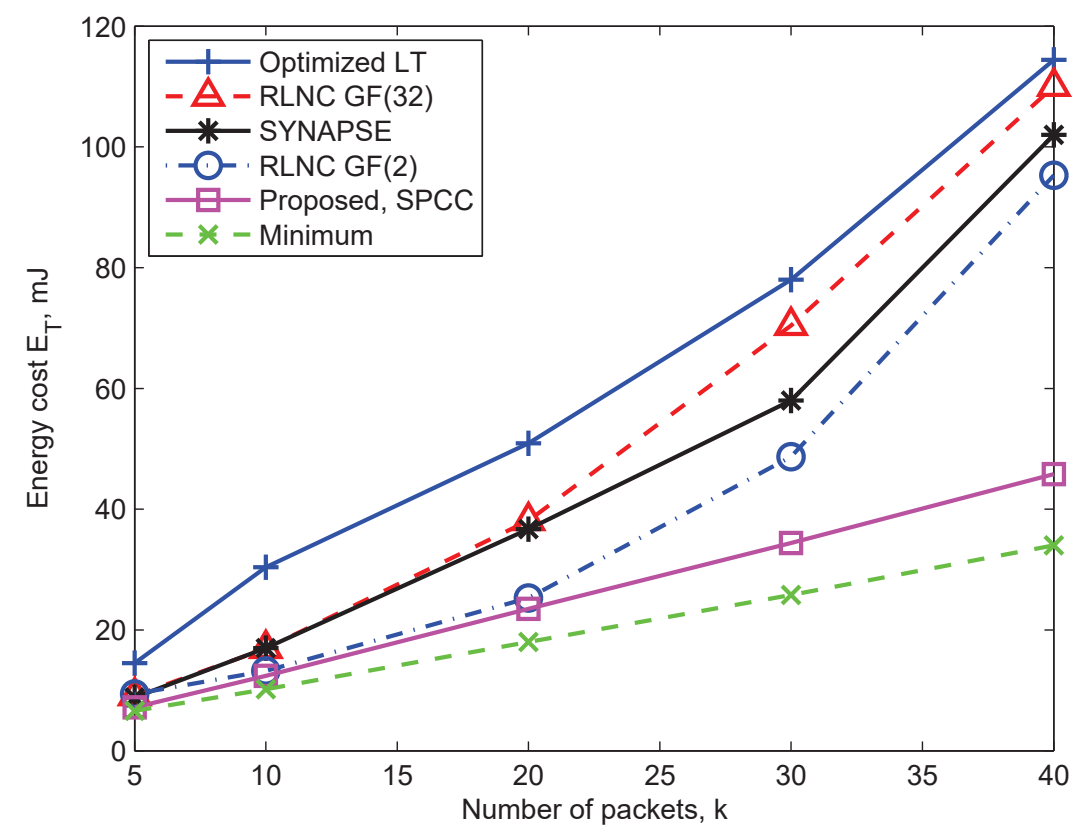

(a) Unicast network with one receiver.

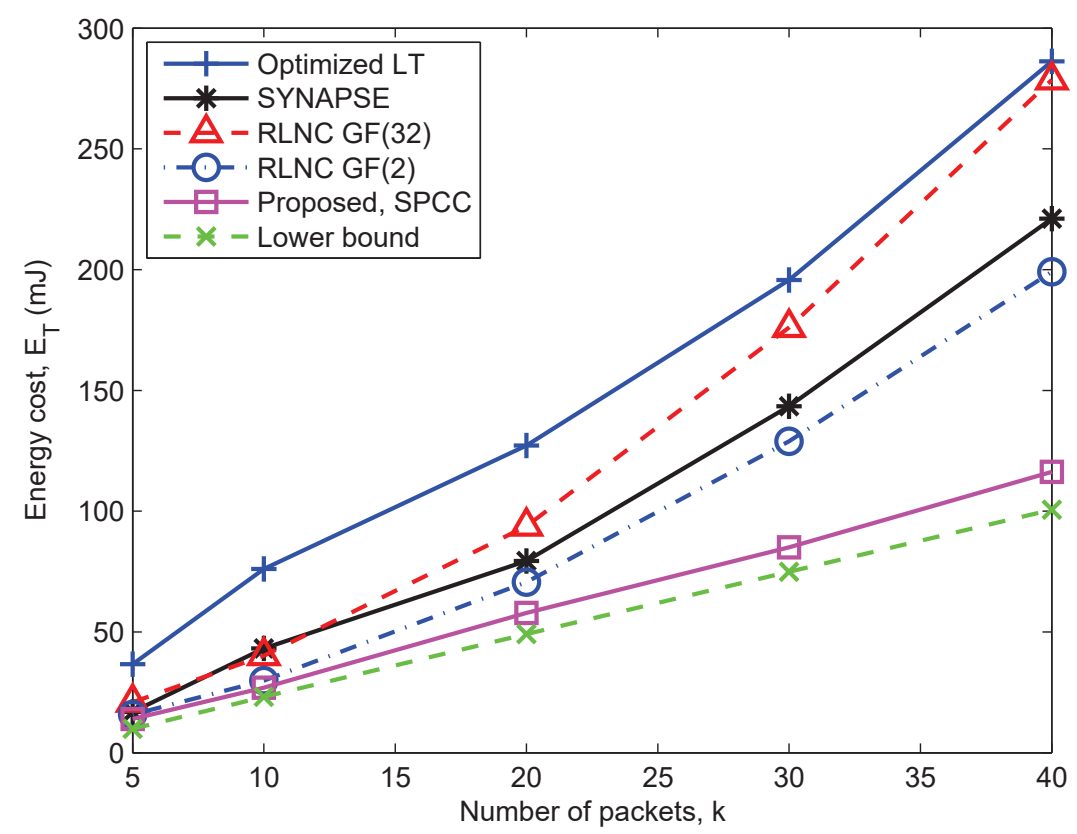

(b) Multicast network with four receivers.

Fig. 9. Total energy cost $E_{T}$ of our proposed coding scheme SPCC, compared with other state of the art erasure coding schemes. 


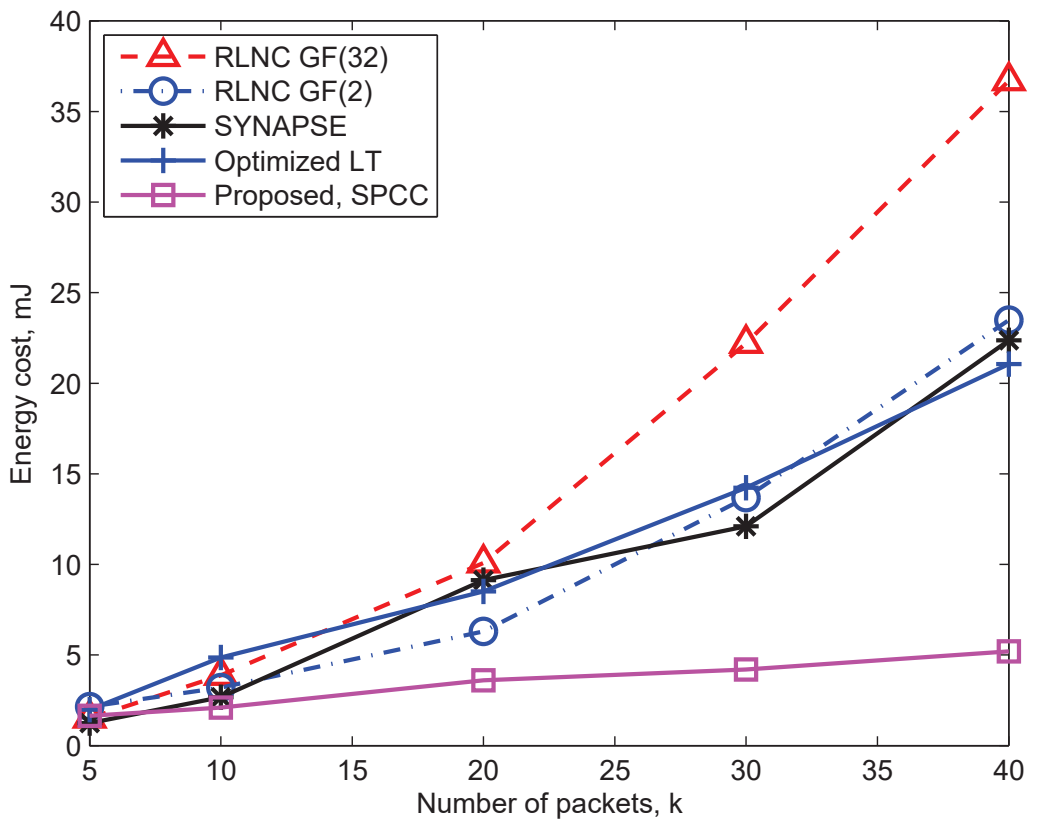

(a) Encoding energy cost at the transmitter.

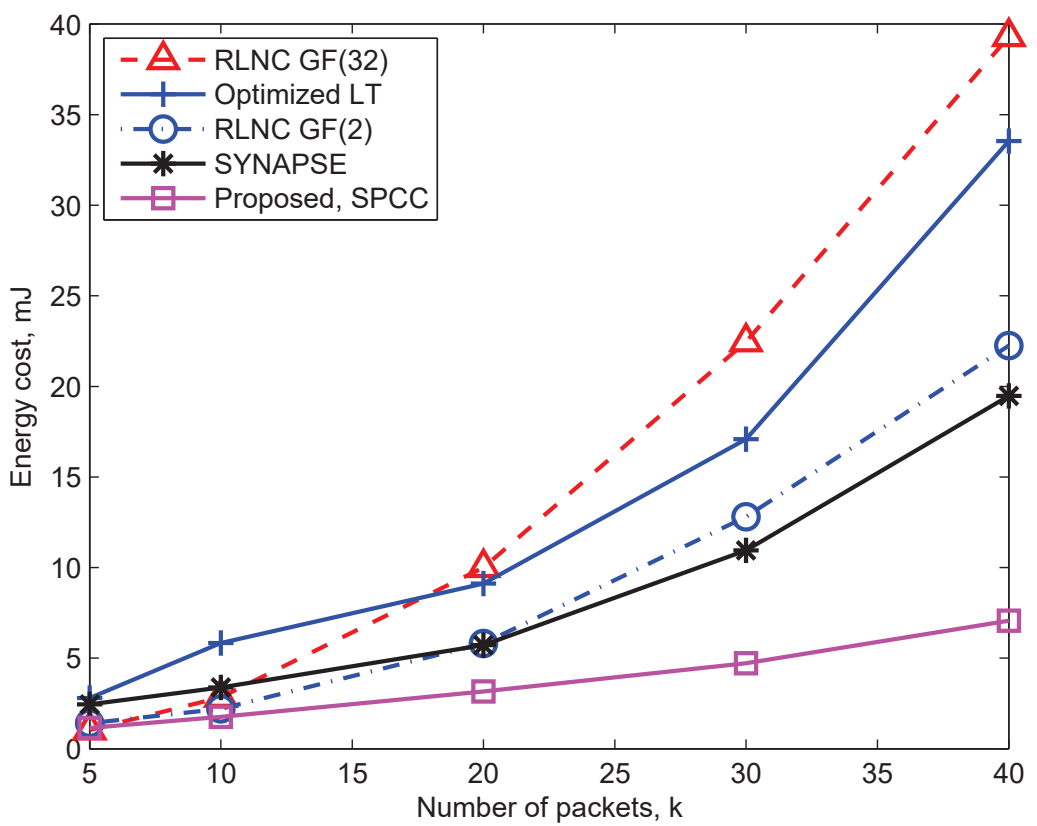

(b) Decoding energy cost for one receiver.

Fig. 10. Energy cost of encoding and decoding. 


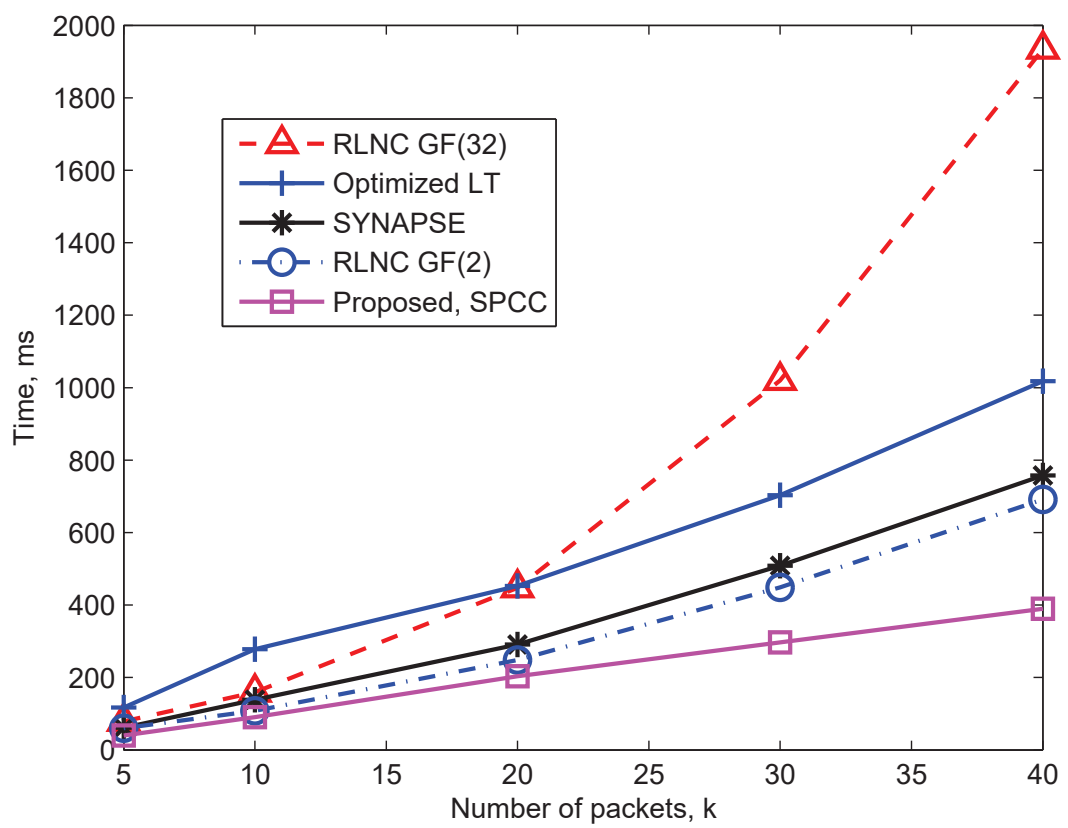

Fig. 11. Average time to receive packets, and for successful decoding. The average value was calculated for a multicast network with four receivers.

over a specific time period, and the duration of the time period. The total energy cost, $E_{T}$, is the sum of energy costs incurred in the transmitter and all $n$ receivers. For the transmitter, its energy cost includes encoding of $E\left[T_{k}\right]$ packets, transmission of $E\left[T_{k}\right]$ packets, reception of $n$ ACK packets. For each receiver, its energy cost includes reception of $E\left[R_{\varphi, k}\right]$ packets, decoding of $E\left[R_{\varphi, k}\right]$ packets, and transmission of an accumulated ACK packet.

We also compare the performance of SPCC with a lower bound on energy cost which we calculate as follow. We assume that energy cost of computation due to encoding and decoding is zero, and $E[\delta]$ is zero. The lower bound represents the case when the channel condition is perfect and thus the packets are transmitted in their raw form. In other words, the lower bound captures the ideal situation where transmissions are always successful and no additional measure to deal with error transmission is implemented.

We plot the energy cost for the lower bound and other schemes in Fig. 9. For each $E_{T}$ value plotted on the graph, we repeated the experiment five times, and then took its average. The results in the figure show that our proposed scheme performs significantly better than the other state of the art erasure coding schemes, and its total energy cost is very close to the lower bound. 
The results also show that such energy gain increases as the value of $k$ increases. For $k=40$, the SPCC reduces the energy cost consumption by more than $70 \%$ and $100 \%$ for the multicast and unicast networks respectively when compared to the next best performing coding scheme, RLNC $G F(2)$.

The results of the graphs shows that while an optimized LT code has linear encodingdecoding computational complexities, such energy gain is mired due to high reception redundancy. Similarly while RLNC over $G F(256)$ has near zero redundancy, the energy gain of minimal transmissions and receptions is adversely affected by high decoding computational complexity. A breakdown of the energy cost consumption of the encoding-decoding operations of various coding schemes is plotted in Fig. 10. The graphs show that for encoding the energy cost increases linearly, whereas for decoding the energy cost increases by a polynomial factor. While SYNAPSE has lower encoding and decoding energy cost compared to RLNC over $G F(2)$ such gains are offset due to higher redundancy, which increases the energy cost of transmissions and receptions.

In Fig. 11, we plot the total time taken for a receiver to receive all the packets and perform successful decoding. The results show that SPCC takes the shortest time duration until successful decoding. The time taken to receive packets is short as SPCC can perform successful decoding with $k$ coded packets with high probability, and due to the use of table based RNG and sparse encoding, the time taken for decoding is also short.

\section{CONCLUSION}

Motivated by the growing popularity of low-powered embedded devices in IoT, and the inherent power-constraint nature of these devices, we studied the problem of reducing the total energy cost of erasure coding scheme in this paper.

The efficiency of transmitting a batch of $k$ packets to $n$ receivers over an erasure channel can be improved by applying erasure codes for transmission. While coding over $G F(2)$ enjoys low computational complexity, it demands high reception redundancy. On the other hand, coding over $G F(32)$ requires high computational complexity, it offers low reception redundancy. We proposed an efficient erasure coding scheme, SPCC, which uses parallel concatenation of codes over different field sizes. SPCC finds a balanced mix between $G F(2)$ and $G F(32)$ coded packets to minimize the energy cost. 
To further reduce the energy cost, we used sparse coding vector and table based method to generate coding vectors. Testbed implementation of SPCC with other state of the art erasure coding schemes showed that SPCC performs significantly better than other coding schemes in terms of energy cost, and such gain in energy cost is additive with increasing packet generation size.

The use of systematic erasure code, where the transmitter first transmits $k$ input packets and then start transmitting coded packets, has shown to reduce the reception redundancy [11]. As part of our future work we would be interested to study how incorporating systematic erasure coding scheme in SPCC can contribute towards reducing the total energy cost.

\section{REFERENCES}

[1] S. Brown, O. Johnson, and A. Tassi, "Reliability of Broadcast Communications Under Sparse Random Linear Network Coding," IEEE Trans. Veh. Technol., vol. 67, no. 5, pp. 4677-4682, May 2018.

[2] E. Casilari, J. M. Cano-Garci, and G. Campos-Garrido, "Modeling of Current Consumption in 802.15.4/ZigBee Sensor Motes," Sensors, vol. 10, no. 6, pp. 5443-5468, 2010.

[3] D. Ferreira, R. A. Costa, and J. Barros, "Real-Time Network Coding for Live Streaming in Hyper-Dense WiFi Spaces," IEEE J. Sel. Areas Commun., vol. 32, no. 4, pp. 773-781, Apr. 2014.

[4] J. B. Fraleigh and R. A. Beauregard, Linear Algebra. Addison-Wesley Publishing Company, 1995, chapter 10.

[5] E. Gossett, Discrete Mathematics with Proof. Wiley, 2009, 2nd Edition.

[6] J.-H. Hauer, V. Handziski, and A. Wolisz, "Experimental Study of the Impact of WLAN Interference on IEEE 802.15.4 Body Area Networks," in European Conf. on Wireless Sensor Networks, Cork, Ireland, Feb. 2009.

[7] J. Heide, M. V. Pedersen, F. H. Fitzek, and T. Larsen, "Cautious View on Network Coding - From Theory to Practice," IEEE J. of Commun. and Networks, vol. 10, no. 4, pp. 403-411, Dec. 2008.

[8] Q. Hu and J. Zheng, "Weight Pick: an efficient packet selection algorithm for network coding based multicast retransmission in mobile communication networks," Springer Wireless Networks, vol. 19, no. 3, pp. 363-372, Apr. 2013.

[9] S. Jafarizadeh and A. Jamalipour, "An exact solution to degree distribution optimization in LT codes," in Wireless Commun. and Networking Conf. (WCNC), 2014 IEEE, Istanbul, Turkey, Apr. 2014.

[10] J. Jin, B. Li, and T. Kong, "Is Random Network Coding Helpful in WiMAX?" in Proc. INFOCOM 2008. The 27th Conf. on Comput. Commun. IEEE, Phoenix, USA, Apr. 2008.

[11] A. L. Jones, I. Chatzigeorgiou, and A. Tassi, "Binary systematic network coding for progressive packet decoding," in Commun. (ICC), 2015 IEEE Int. Conf. on, London, UK, Sept. 2015.

[12] D. Miorandi, S. Sicari, F. D. Pellegrini, and I. Chlamtac, "Internet of things: Vision, applications and research challenges," Ad Hoc Networks, vol. 10, no. 7, pp. 1497 - 1516, Sept. 2012.

[13] M. Nistor, D. Lucani, and J. Barros, "A Total Energy Approach to Protocol Design in Coded Wireless Sensor Networks," in Proc. Int. Symp. on Network Coding (NetCod), Cambridge, USA, June 2012.

[14] P. Ostovari and J. Wu, "Reliable Broadcast with Joint Forward Error Correction and Erasure Codes in Wireless Communication Networks," in Proc. Mobile Ad Hoc and Sensor Systems (MASS), Dallas, USA, Oct. 2015.

[15] K. Philips, "Ultra Low Power short range radios: Covering the last mile of the IoT," in European Solid State Circuits Conf. (ESSCIRC), Venice Lido, Italy, Sept. 2014. 
[16] J. Qureshi, C. H. Foh, and J. Cai, "Primer and Recent Developments on Fountain Codes," Recent Advances in Commun. and Networking Technol., vol. 2, no. 1, pp. 2-11, July 2013.

[17] M. Rossi, N. Bui, G. Zanca, L. Stabellini, R. Crepaldi, and M. Zorzi, "SYNAPSE++: Code Dissemination in Wireless Sensor Networks Using Fountain Codes,” IEEE Trans. on Mobile Comput., vol. 9, no. 12, pp. 1749-1765, Dec. 2010.

[18] J. K. Sundararajan, D. Shah, M. Medard, S. Jakubczak, M. Mitzenmacher, and J. Barros, "Network Coding Meets TCP: Theory and Implementation,” Proceedings of the IEEE, vol. 99, no. 3, pp. 490-512, Mar. 2011.

[19] P. Suriyachai, U. Roedig, and A. Scott, "Implementation of a MAC Protocol for QoS Support in Wireless Sensor Networks," in Proc. IEEE Int. Conf. on Pervasive Comput. and Commun., Galveston, USA, Mar. 2009.

[20] A. Tassi, I. Chatzigeorgiou, and D. Lucani, "Analysis and Optimization of Sparse Random Linear Network Coding for Reliable Multicast Services,” IEEE Trans. on Commun., vol. 64, no. 1, pp. 285-299, Jan. 2016.

[21] O. Trullols-Cruces, J. Barcelo-Ordinas, and M. Fiore, "Exact Decoding Probability Under Random Linear Network Coding," IEEE Commun. Lett., vol. 15, no. 1, pp. 67 - 69, Jan. 2011.

[22] W. Yadong and D. Shihong, "Link Layer Time-Varying Model for IEEE 802.15.4 Radio in Industrial Environment," Int. J. of Distributed Sensor Networks, 2014. 